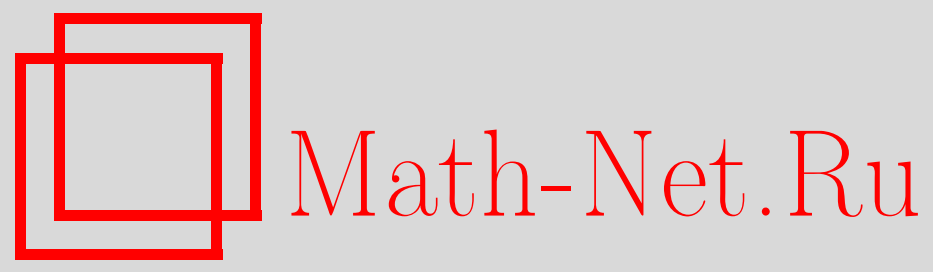

А. Ф. Ронжин, Расширения информационных протоколов, основанных на отображениях конечных множеств, Дискрет. матем., 2005, том 17, выпуск 4, 18-28

DOI: https://doi.org/10.4213/dm126

Использование Общероссийского математического портала Math-Net.Ru подразумевает, что вы прочитали и согласны с пользовательским соглашением http: //www . mathnet.ru/rus/agreement

Параметры загрузки:

IP : 35.174 .16 .151

26 апреля 2023 г., 06:36:48

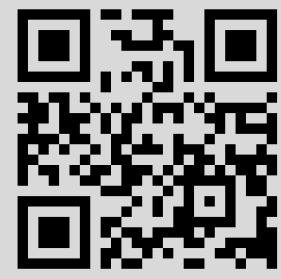


УДК 519.7

\title{
Расширения информационных протоколов, основанных на отображениях конечных множеств
}

( 2005 г. А. Ф. Ронжин

\begin{abstract}
Вводятся понятия информационного протокола на конечном множестве, его расширения и разложения. Приводятся примеры махинаций злоумышленника с информационными протоколами. Предлагается система защиты информационных протоколов, допускающих разложение на большое количество расширений, и вычисляются некоторые вероятностные характеристики ее качества.
\end{abstract}

\section{1. Определения и основные свойства}

Зафиксируем конечное непустое множество $S$. Пусть $C(S)$ - множество всех отображений множества $S$ в себя. Далее всюду полагаем, что $\varnothing$ - пустое множество, для любого подмножества $X \subset S$ и отношения $\rho \in C(S)$ запись $|X|$ обозначает мошность множества $X, \rho(X)$ и $\rho^{-1}(X)-$ образ и прообраз множества $X$, соответственно.

Пару отображений $(\varphi, \psi)$, где $\varphi, \psi \in C(S)$, будем называть информационным протоколом на $S$ (далее всюду протоколом). Протокол $(\varphi, \psi)$ будем называть прозрачным, если $|\varphi(S)|=|\psi(\varphi(S))|$, и мутным, если $|\varphi(S)|>|\psi(\varphi(S))|$.

Протокол $\left(\varphi_{r}, \psi_{r}\right)$ будем называть расширением протокола $(\varphi, \psi)$, если

$$
\begin{aligned}
\varphi_{r}(S) & \subset \varphi(S), \\
\psi\left(\varphi_{r}(s)\right) & =\psi(\varphi(s)) \text { для всех } s \in S .
\end{aligned}
$$

Расширение $\left(\varphi_{r}, \psi_{r}\right)$ протокола $(\varphi, \psi)$ будем называть строгим, если

$$
\left|\psi_{r}\left(\varphi_{r}(S)\right)\right|=|\psi(\varphi(S))|
$$

и противоречивым, если

$$
\left|\psi_{r}\left(\varphi_{r}(S)\right)\right|>|\psi(\varphi(S))|
$$

Из соотношений (1) и (2) следует, что для расширения $\left(\varphi_{r}, \psi_{r}\right)$ справедливы неравенства

$$
|S| \geqslant|\varphi(S)| \geqslant\left|\left(\varphi_{r}(S)\right)\right| \geqslant|\psi(\varphi(S))| \geqslant 1 .
$$


Для противоречивого расширения из (3) и (4) следует, что

$$
|S| \geqslant|\varphi(S)| \geqslant\left|\left(\varphi_{r}(S)\right)\right| \geqslant\left|\psi_{r}\left(\varphi_{r}(S)\right)\right|>|\psi(\varphi(S))| \geqslant 1 .
$$

Семейство расширений протокола $(\varphi, \psi)$

$$
R(\varphi, \psi)=\left\{\left(\varphi_{r}, \psi_{r}\right), r \in R\right\}
$$

где $R$ - конечное множество, назовем разложением протокола $(\varphi, \psi)$, если

$$
\varphi(S)=\bigcup_{r \in R} \varphi_{r}(S), \quad \varphi_{r_{1}}(S) \cap \varphi_{r_{2}}(S)=\varnothing, \quad r_{1} \neq r_{2} .
$$

Разложение $R(\varphi, \psi)$ назовем регулярным, если $\left|\varphi_{r}(S)\right|=|\psi(\varphi(S))|$ для всех $r \in R$.

Кроме того, пусть $\xi$ - некоторая случайная величина с значениями на $S$, вероятностями $\mathbf{P}\{\xi=s\}>0, s \in S$, и

$$
\mathbf{P}_{\varphi, \psi}(x, y)=\mathbf{P}_{\varphi, \psi}\{\varphi(\xi)=x, \psi(\varphi(\xi))=y\}
$$

- совместное распределение пары случайных величин $(\varphi(\xi), \psi(\varphi(\xi)), x \in \varphi(S)$, $y \in \psi(\varphi(S))$. Очевидно, что $\mathbf{P}_{\varphi, \psi}(x, y)=\mathbf{P}\left\{\varphi(\xi) \in \varphi^{-1}(x)\right\}$, если $y=\psi(x)$, и 0 в противном случае.

По аналогии с [1] введем обозначения

$$
\begin{aligned}
& \mathbf{P}_{\psi}(y)=\sum_{x \in \varphi(S)} \mathbf{P}_{\varphi, \psi}(x, y), \\
& \mathbf{P}_{\psi}(x)=\sum_{y \in \psi(\varphi(S))} \mathbf{P}_{\varphi, \psi}(x, y),
\end{aligned}
$$

и определим среднюю взаимную информацию протокола $(\varphi, \psi)$ как величину

$$
\mathbf{I}(\varphi, \psi)=\sum_{x, y} \mathbf{P}_{\varphi, \psi}(x, y) \ln \left(\mathbf{P}_{\varphi, \psi}(x, y) / \mathbf{P}_{\psi}(y) \mathbf{P}_{\varphi}(x)\right)
$$

Вычисления показывают, что

$$
\mathbf{P}_{\varphi}(x)=\mathbf{P}\{\varphi(\xi)=x\}, \quad \mathbf{P}_{\psi}(y)=\mathbf{P}\{\psi(\varphi(\xi))=y\}
$$

и

$$
\mathbf{I}(\varphi, \psi)=-\sum_{y \in \psi(\varphi(S))} \mathbf{P}_{\psi}(y) \ln \mathbf{P}_{\psi}(y)
$$

Для $\mathbf{I}(\varphi, \psi)$ справедливы неравенства

$$
0 \leqslant \mathbf{I}(\varphi, \psi) \leqslant-\sum_{x \in \varphi(S)} \mathbf{P}\{\varphi(\xi)=x\} \ln \mathbf{P}\{\varphi(\xi)=x\},
$$

причем слева равенство достигается тогда и только тогда, когда

$$
|\psi(\varphi(S))|=1,
$$

а равенство справа тогда и только тогда, когда протокол $(\varphi, \psi)$ прозрачный. 
Теорема 1 (теорема расширения). Протокол $(\varphi, \psi)$ имеет противоречивое расширение $\left(\varphi_{r}, \psi_{r}\right)$ тогда и только тогда, когда он мутный. При этом существует его расширение $\left(\varphi_{r}, \psi_{r}\right)$ такое, что средня взаимная информачия $\mathbf{I}(\varphi, \psi)$ меньше средней взаимной информачии этого расширения $\mathbf{I}\left(\varphi_{r}, \psi_{r}\right)$.

Доказательство. Необходимость условий утверждения следует из соотношений (5).

Докажем достаточность. Пусть протокол $(\varphi, \psi)$ является мутным. Существуют $y_{1}, y_{2}, \ldots, y_{k} \in \varphi(S)$, где $k \geqslant 2$, такие, что $\psi\left(y_{1}\right)=\ldots=\psi\left(y_{k}\right)=z_{1}$, и существуют $z_{2}, \ldots, z_{k} \in \varphi(S) \backslash \psi(\varphi(S))$. Пусть $\psi_{r}\left(y_{j}\right)=z_{j}$ для всех $j=1, \ldots, k$ и и $\psi_{r}(s)=\psi(s)$, если $s \in S \backslash\left\{y_{1}, \ldots, y_{k}\right\}$.

Очевидно, что

$$
\left|\psi_{r}\left(\varphi_{r}(S)\right)\right|=|\psi(\varphi(S))|+k-1>|\psi(\varphi(S))| .
$$

Это неравенство завершает доказательство достаточности условий теоремы.

Средняя взаимная информация протокола $\left(\varphi_{r}, \psi_{r}\right)$ вычисляется по формуле

$$
\begin{array}{r}
\mathbf{I}\left(\varphi_{r}, \psi_{r}\right)=\mathbf{I}(\varphi, \psi)+\mathbf{P}\left\{\psi(\xi) \in\left\{y_{1}, y_{2}, \ldots, y_{k}\right\}\right\} \ln \mathbf{P}\left\{\psi(\xi) \in\left\{y_{1}, y_{2}, \ldots, y_{k}\right\}\right\} \\
+\sum_{i=1}^{k}\left(-\mathbf{P}\left\{\varphi(\xi)=y_{i}\right\} \ln \mathbf{P}\left\{\varphi(\xi)=y_{i}\right\}\right) .
\end{array}
$$

Сумма последних двух слагаемых положительна.

Доказательство теоремы завершено.

Следствие 1. Любой протокол имеет расширение, являющееся прозрачным.

Теорема 2 (теорема разложения). Протокол $(\varphi, \psi)$ имеет разложение на $m$ расиирений тогда и только тогда, когда

$$
m \leqslant \min _{y \in \psi(\varphi(S))}\left|\psi^{-1}(y) \cap \varphi(S)\right| .
$$

Доказательство. Пусть существует разложение $(\varphi, \psi)$ на $m$ расширений. Тогда для всех $s$ из $S$

$$
\psi(\varphi(s))=\psi\left(\varphi_{r_{1}}(s)\right)=\cdots=\psi\left(\varphi_{r_{m}}(s)\right), \quad r_{i} \in R, \quad i=1, \ldots, m .
$$

В силу (6) для всех $s$ из $S$ и $i \neq j$

$$
\varphi_{r_{i}}(s) \neq \varphi_{r_{j}}(s)
$$

Пусть $y_{m} \in \psi(\varphi(S))$ таково, что

$$
\left|\psi^{-1}\left(y_{m}\right) \cap \varphi(S)\right|=\min _{y \in \psi(\varphi(S))}\left|\psi^{-1}(y) \cap \varphi(S)\right| .
$$

Тогда в $y_{m}$ переходят не более, чем $\left|\psi^{-1}\left(y_{m}\right)\right|$ элементов из $\varphi(S)$, следовательно, среди $\varphi_{r_{1}}(s), \ldots, \varphi_{r_{m}}(s)$ при $s \in \varphi^{-1}\left(\psi^{-1}\left(y_{m}\right)\right)$ не более $\left|\psi^{-1}\left(y_{m}\right)\right|$ различных элементов из $S$, и для выполнения (10) необходимо выполнение условия

$$
m \leqslant\left|\psi^{-1}\left(y_{m}\right)\right| \text {. }
$$


Необходимость доказана.

Докажем достаточность. Пусть

$$
\begin{gathered}
|\psi(\varphi(S))|=\left\{y_{1}, \ldots, y_{k}\right\}, \quad m_{i}=\left|\psi^{-1}\left(y_{i}\right) \cap \varphi(S)\right|, \\
X_{i}=\left\{x_{i, 1} \ldots, x_{i, m_{i}}\right\}=\psi^{-1}\left(y_{i}\right) \cap \varphi(S), \quad i=1, \ldots, k, \quad m \leqslant \min _{i} m_{i} .
\end{gathered}
$$

Определим отображения $\varphi_{r}(s)=x_{i, r}, \psi_{r}(s)=s$, если $\varphi(s) \in X_{i}, r=1, \ldots, m$. Из построения видно, что $\left(\varphi_{r}, \psi_{r}\right)$ является расширением $(\varphi, \psi)$ и $\psi_{r_{i}}(S) \cap \psi_{r_{j}}(S)=\varnothing$ при $i \neq j$. Если $\varphi_{1}(S) \cup \ldots \cup \varphi_{m}(S)=\varphi(S)$, то утверждение доказано. Это означает, что $m_{1}=\ldots=m_{k}=m=|\varphi(S)| /|\psi(\varphi(S))|$, и построенное разложение является регулярным.

В противном случае построим протокол $\left(\varphi_{0}, \psi_{0}\right)$ следующим образом. Обозначим через $X$ все элементы $\varphi(S)$, не вошедшие в $\varphi_{1}(S) \cup \ldots \cup \varphi_{m}(S)$. Положим $\varphi_{0}(s)=\varphi(s)$, если $s$ принадлежит $\varphi^{-1}(X)$, и $\varphi_{0}(s)=\varphi_{m}(s)$, если $s$ не принадлежит $\varphi^{-1}(X), \psi_{0}(s)=s$. По построению $\left(\varphi_{0}, \psi_{0}\right)$ является расширением $(\varphi, \psi), \varphi_{0}(S) \cup \ldots \cup \varphi_{m-1}(S)=\varphi(S)$, и значит, $\left\{\left(\varphi_{r}, \psi_{r}\right), r=0,1, \ldots, m-1\right\}$ является требуемым разложением.

Теорема доказана.

\section{2. Примеры махинаций в мутных протоколах}

Прикладной смысл данного в предыдущем пункте определения протокола применительно к информационным системам заключается в следующем.

Система информацию о своем состоянии $S$ из $S$ передает участнику системы в виде $\varphi(s)$, который, в свою очередь, принимает некоторое решение $\psi(\varphi(s))$. Использование мутных протоколов может привести к нарушению безопасности системы посредством действий злоумышленника (нарушителя безопасности системы), находящегося в системе, возможно в сговоре с сообщником (агентом), находящимся у участника системы.

К примерам таких нарушений можно отнести махинации в системах нотариальной заверки цифровых подписей [2], организации скрытых [3], потайных [4] каналов для обмена данными между злоумышленником и сообщником.

\section{1. Махинации в системах нотариальной заверки цифровых подписей}

Рассматривается сеть передачи сообщений $x$ из конечного множества всех сообщений $X$ между абонентами из множества $I=\{0,1, \ldots, N\}, N>2$. Абонент 0 объявлен нотариусом. Каждый абонент $i$ имеет набор секретных функций $d_{i, j}(x), x \in X$, которые принимают значения из множества $D, j \in I$. Они используются абонентом $i$ для создания цифровой подписи сообщений $x$ абонентам $j$. Кроме того, $i$ имеет набор секретных функций $b_{j, i}(x, d), x \in X, d \in D$, которые принимают значение из множества $D, j \in I$. Они используются абонентом $i$ для проверки правильности цифровой подписи $d$ под сообщением $x$ от абонента $j$. Абонент $i \in\{1, \ldots, N\}$ знает только функщии $d_{i, j}(x)$, $b_{j, i}(x, d), j \in I, d \in D$. Нотариус знает все функщия. Получив от абонента $i$ вектор $(i, j, x, d)$ нотариус создает вектор $s=\left(i, j, x, d, b_{i, j}(x, d)\right)$ и через сеть передает его абоненту $j$. Обратим внимание на то, что функция $b_{i, j}(x, d)$ известна лишь нотариусу и абоненту $j$. Абоненту $i$ она неизвестна. 
Абонент $j$, получив через сеть вектор $s=(i, j, x, d, b)$, принимает решение о том, что сообщение $x$ с подписью $d$ подписано правильно, если вычисленное им значение $b_{i, j}(x, d)$ совпадает с $b$, и отображает его в себя. В противном случае, считает его неподписанным и отображает в вектор $(i, i, x, d, b)$.

Выше описан протокол $(\varphi, \psi)$ для общения системы с участником $j$, в котором

$$
S=\{(i, j, x, d, b)\}, \quad i \in I, \quad x \in X, \quad d \in D, \quad b \in B, \quad \varphi(s)=s,
$$

$\psi(s)=s$, если $b_{i, j}(x, d)=b$, и $\psi(s)=(i, i, x, d, b)$, если $b_{i, j}(x, d) \neq b$.

Одна из функщй механизма электронной цифровой подписи - это разрешение конфликта, в котором абонент $j$ утверждает, что получил от $i$ сообщение $x$, подписанное подписью $d$, а абонент $i$ от этого отказывается. Обычно, условием разрешения конфликта в пользу абонента $i$ является справедливость неравенства $d_{i, j}(x) \neq d$.

Пусть $S_{0}$ - множество тех состояний системы из $S$, для которых соответствующие им сообщения $x$ приняты абонентом $j$ как подписанные правильно.

Рассмотрим ограничение описанного выше протокола на множество $S_{\mathbf{0}}$.

Из приведенных выше определений видно, что для всех $x$

$$
s_{u}=\left(i, j, x, d_{i, j}(x), b_{i, j}\left(x, d_{i, j}(x)\right)\right) \in \psi\left(\varphi\left(S_{0}\right)\right) .
$$

Пусть для некоторых $i, x$ эффективно вычислены при неизвестных функщия $b_{i, j}$, $b_{i_{1}, j}$ такие $i_{1}, x_{1}, d_{1}$, что

$$
b_{i, j}\left(x, d_{i, j}(x)\right)=b_{i_{1}, j}\left(x_{1}, d_{1}\right),\left(i_{1}, x_{1}, d_{1}\right) \neq\left(i, x, d_{i, j}(x)\right) .
$$

В силу условия (11) абонент $j$ примет решение о том, что сообщение $x_{1}$ от абонента $i_{1}$ с подписью $d_{1}$ подписано правильно, то есть

$$
\psi\left(\varphi\left(s_{f}\right)\right)=S_{f}
$$

В случае возникновения конфликта абонент $i_{1}$ будет отказываться от сообщения $x_{1} \mathrm{c}$ подписью $d_{1}$. Он сможет это сделать, если $d_{i, j}\left(x_{1}\right) \neq d_{1}$.

Замечание 1. Пусть функщия $b_{i, j}(x, d)$ не зависит от $d$. Тогда любой абонент $i_{1}$ откажется от любого сообщения $x_{1}$ с подписью $d_{1} \neq d_{i_{1}, j}\left(x_{1}\right)$, проведя описанную выше махинацию при $i=i_{1}, x=x_{1}$, если подменит

$$
S_{n}=\left(i_{1}, j, x_{1}, d_{i_{1}, j}\left(x_{1}\right), b_{i_{1}, j}\left(x_{1}\right)\right)
$$

вектором

$$
S_{f}=\left(i_{1}, j, x_{1}, d_{1}, b_{i_{1}, j}\left(x_{1}\right)\right) .
$$

Замечание 2. Пусть функция $b_{i, j}(x, d)$ не зависит от $i$. Тогда любой абонент $i_{1}$ откажется от любого сообщения $x_{1}$ с подписью $d_{1}=d_{i, j}\left(x_{1}\right) \neq d_{i_{1}, j}(x)$, проведя описанную выше махинацию при $x=x_{1}$ в сговоре с абонентом $i$.

\section{2. Потайные, скрытые каналы}

Пусть протокол $(\varphi, \psi)$ имеет разложение (6). Тогда злоумышленник (возможно, это программно-аппаратная закладка) может организовать канал передачи команд своему сообнику (возможно, программно-аппаратной закладке), находящемуся у участника, следующим образом. 
Не ограничивая общности, полагаем $R \subset S$.

Пусть злоумышленник желает передать сообщнику команду с именем $r$. Тогда, при передаче системой участнику сообщения о своем состоянии $\varphi(s)$, злоумышленник подменяет $\varphi(s)$ на $\varphi_{r}(s)$. В силу условий (1) и (2) на расширения $\left(\varphi_{r}, \psi_{r}\right)$ участник не заметит такой подмены. Сообщник же примет решение об исполнения команды с именем $r$ на том основании, что полученное им сообщение $\varphi_{r}(s)$ принадлежит множеству $\varphi_{r}(S)$. В силу условий (6) его решение будет однозначным и правильным. Конечно, такая подмена имеет смысл лишь в случае $|R| \geqslant 2$.

Далее рассмотрим довольно общий пример, встречающихся в практике ситуаций. На конечном промежутке времени $T$ в фиксированные моменты времени в системе происходят события с признаками $0,1, \ldots, N$. Например, передаются сообщения со значениями атрибута $0,1, \ldots, N$ (см. [5]). Система передает информацию о событиях своему участнику. Информация о характере события (сообщения) недоступна никому, кроме системы и участника, а вот признаки (значения атрибутов) наблюдаемы. Рассмотрим в качестве модели наблюдений за передачей признаков событий выборку без возвращений.

Пусть множеством состояний системы $S$ является множество выборов без возвращения из урны с $n+h_{0}$ шарами, содержащей $h_{i} \geqslant 1$ шаров цвета $i, i=0,1, \ldots, N$, где $h_{1}+\ldots+h_{N}=n$. Будем обозначать это множество $S\left(h_{0}, \ldots, h_{N}\right)$, а его элементы

$$
s=\left(x_{1}, \ldots, x_{n+h_{0}}\right), \quad x_{j}=0,1, \ldots, N, \quad j=1, \ldots, n+h_{0} .
$$

Решения участника могут не зависеть от порядка следования всех событий или событий с некоторыми признаками. Поэтому для дальнейших обсуждений определим еще ряд множеств. Пусть

$I\left(n, h_{0}\right)$ - множество всех наборов целых чисел $i=\left(i_{1}, \ldots, i_{n}\right)$ таких, что $1 \leqslant i_{1}<\ldots<$ $i_{n} \leqslant n+h_{0}$

$S\left(h_{1}, \ldots, h_{N}\right)$ - множество всех выборов без возвращения из урны с $n$ шарами, содержащей $h_{i} \geqslant 1$ шаров цвета $i, i=1, \ldots, N$, которые будем обозначать $a=$ $\left(a_{1}, \ldots, a_{n}, a_{j}=1, \ldots, N, j=1, \ldots, n\right)$;

$T(i), i \in I\left(n, h_{0}\right)$ - это множество всех элементов $S\left(h_{1}, \ldots, h_{N}\right)$, у которых на местах $i$ не стоит элемент цвета 0 ;

$P(a), a \in S\left(h_{1}, \ldots, h_{N}\right)$ - множество всех элементов $S\left(h_{0}, \ldots, h_{N}\right)$, у которых есть подпоследовательность $a$, то есть существует $i \in I\left(n, h_{0}\right)$ такое, что $x_{i_{v}}=a_{\nu}$, $v=1, \ldots, n$.

Для $s \in S$ определим следующие отображения:

$\varepsilon(s)=s$ отображает элемент $s$ в себя;

$\tau_{t}(s)=t$ отображает все элементы $S$ в фиксированный элемент $t \in S$;

$\mu_{i}(s)$ отображает $s$ в элемент, у которого шары цветов $1, \ldots, N$ стоят в том же порядке, как в $s$, но на местах $i \in I\left(n, h_{0}\right)$;

$\pi_{a}(s)$ отображает $s$ в элемент, у которого на тех местах, где стояли шары цветов $1, \ldots, N$, стоит последовательность шаров $a \in S\left(h_{1}, \ldots, h_{N}\right)$;

$\chi(s)$ принимает фиксированное значение на всех элементах множества $S$ (это значит, что участнику важен лишь факт передачи информации о всех событиях за период $T$ ); 
$\omega(s)$ принимает одно фиксированное значение на всех элементах множества $P_{a}, a \in$ $S\left(h_{1}, \ldots, h_{N}\right)$, причем, разные значения при разных $a$ (это означает, что участнику важен лишь порядок, в котором следуют события с признаками $1, \ldots, N)$;

$\lambda(s)$ принимает одно фиксированное значение на всех элементах множества $T(i), i \in$ $I\left(n, h_{0}\right)$, причем, разные значения при разных $i$ (это означает, что участнику важно лишь, в какие моменты времени происходили события с признаками $1, \ldots, N)$.

Теорема 3. Семейство протоколов $\left\{\left(\tau_{t}, \varepsilon\right), t \in S\right\}$ является регулярньм разложением протокола $(\varepsilon, \chi)$ на $\left(n+h_{0}\right) ! /\left(h_{0} ! \ldots h_{n} !\right)$ расширений.

Семейство протоколов $\left\{\left(\mu_{i}, \varepsilon\right), i \in I\left(n, h_{0}\right)\right\}$ является регулярным разложением протокола $(\varepsilon, \omega)$ на $\left(n+h_{0}\right) ! /\left(h_{0} ! n !\right)$ расширений.

Семейство протоколов $\left\{\left(\pi_{a}, \varepsilon\right), a \in S\left(h_{1}, \ldots, h_{N}\right)\right\}$ является регулярным разложением протокола $(\varepsilon, \lambda)$ на $n ! /\left(h_{1} ! \ldots h_{N} !\right)$ расширений.

Справедливость утверждений теоремы следует из построения соответствующих отображений.

\section{3. Использование расширений информационных}

\section{протоколов для их защиты}

По-видимому, наилучший способ не допускать нарушения безопасности информационных систем - это использовать только прозрачные протоколы. Однако, это не всегда возможно в связи с наличием таких требований к информационным системам как оперативность и ограниченность технических ресурсов.

Одним из возможных подходов к решению задач безопасности использования мутных протоколов, допускающих разложение на большое количество расширений, является следующий.

Пусть $(\varphi, \psi)$ допускает разложение (6) и $Q-$ подмножество $R$. Тогда, перед входом участника используется программно-техническое средство безопасности, которое случайным образом выбирает элемент $r$ из $Q$ и осуществляет преобразование его входа при помощи $\varphi_{r}$.

Рассмотрим далее лишь те протоколы $(\varphi, \psi)$, у которых отображения $\varphi$ удовлетворяют условию

$$
\varphi(\varphi(s))=\varphi(s), \quad s \in S
$$

Такое ограничение логично, так как подготовленное для передачи состояние не имеет смысла еще раз изменять. Кроме того, для произвольного протокола $(\varphi, \psi)$ существует взаимно однозначное отображение (подстановка) $\pi$ множества $S$ на себя такое, что отображение $\pi(\varphi(s))$ удовлетворяет условию $(12)$, и $(\pi(\varphi(s))), \psi\left(\pi^{-1}(s)\right)$ эквивалентен протоколу $(\varphi, \psi)$ с точки зрения конечных пользователей.

Введем обозначения

$$
\begin{gathered}
\left\{y_{1}, \ldots, y_{m}\right\}=\psi(\varphi(S)), \quad W_{j}=\varphi^{-1}\left(\psi^{-1}\left(y_{j}\right)\right), \\
V_{j}=\psi^{-1}\left(y_{j}\right) \cap \varphi(S), \quad j=1, \ldots, m
\end{gathered}
$$


Лемма 1. Пусть для протокола $(\varphi, \psi)$ выполнено условие (12). Тогда протокол $\left(\varphi_{r}, \psi_{r}\right)$ является расширением протокола $(\varphi, \psi)$ тогда и только тогда, когда для всех $j=$ $1, \ldots, m$

$$
\varphi_{r}\left(W_{j}\right) \subset V_{j}
$$

$\Pi р и$ этом $\varphi_{r}\left(V_{j}\right) \subset V_{j}$

Доказательство. Из условия (12) следует,что

$$
S=W_{1} \cup \ldots \cup W_{m}, \quad \varphi(S)=V_{1} \cup \ldots \cup V_{m}
$$

являются такими разбиениями на непересекающиеся множества, что $W_{j} \supset V_{j}, j=$ $1, \ldots, m$. Пусть $\left(\varphi_{r}, \psi_{r}\right)$ является расширением и $s \in W_{j}, j=1, \ldots, m$. Тогда $\varphi(s) \in V_{j}$, $\varphi_{r}(s) \in \varphi(S), \psi\left(\varphi_{r}(s)\right)=\psi\left(\varphi_{r}(s)\right)=y_{j}$, и следовательно, $\varphi_{r}(s) \in V_{j}$. Необходимость доказана. Достаточность доказывается проверкой условий (1) и (2).

Разложение

$$
\left\{\left(\xi_{z}, \zeta_{z}\right), z \in Z\right\}, \quad \varphi(S)=\bigcup_{z \in Z} \xi_{z}(S), \quad \xi_{z_{1}}(s) \cap \xi_{z_{2}}(s)=\varnothing, \quad z_{1} \neq z_{2},
$$

протокола $(\varphi, \psi)$ будем называть вложенным по отношению к разложению (6), если выполнение условия $\xi_{z}(S) \cap \phi_{r}(S) \neq \varnothing$ влечет выполнение условия $\xi_{z}(S) \supset \phi_{r}(S)$.

Замечание 3. Если разложение (6) регулярное и $|\psi(\varphi(S))|=1$, то любое разложение протокола $(\varphi, \psi)$ будет вложенным по отношению к разложению (6).

Замечание 4. Пусть $S=S\left(h_{0}, h_{1}, \ldots, h_{N}\right),(\varphi, \psi)=(\varepsilon, \omega),\left(\varphi_{r}, \psi_{r}\right)=\left(\mu_{i}, \varepsilon\right)$ (см. теорему 3) и в разложении (13) $\xi_{z}(S)$ переставляет все шары из $T(i), i \in I\left(n, h_{0}\right)$, одним и тем же способом, тогда разложение (13) является вложенным по отношению к $(\varepsilon, \omega)$.

Теорема 4. Пусть протокол $(\varphi, \psi)$ имеет разложения (6) и (13), отображение $\varphi$ удовлетворяет условию (12), $\rho$ - некоторая случайная величина, принимаючая значения из множества $R$.

Тогда для любых $s \in S, z \in Z$ справедливы соотношения

$$
\begin{aligned}
& \mathbf{P}\left\{\rho \in\left\{r: \psi\left(\varphi_{r}(\varphi(s))\right)=\psi(\varphi(s))\right\}\right\}=1 \\
& \mathbf{P}\left\{\rho \in\left\{r: \psi\left(\varphi_{r}\left(\xi_{z}(s)\right)\right)=\psi(\varphi(s))\right\}\right\}=1
\end{aligned}
$$

Пусть, кроме того, разложение (13) является вложенным по отношению к разложению (6).

Тогда существуют такие множества $R(z) \subset R, z \in Z$, что $R\left(z_{1}\right) \cap R\left(z_{2}\right)=\varnothing$ при $z_{1} \neq z_{2} u$

$$
R=\bigcup_{z \in Z} R(z), \xi_{z}(S)=\bigcup_{r \in R(z)} \varphi_{r}(S)
$$

и для любых $s \in S, z_{1}, z_{2} \in Z$ справедливо равенство

$$
\mathbf{P}\left\{\rho \in\left\{r: \varphi_{r}\left(\xi_{z_{1}}(s)\right) \in \xi_{z_{2}}(S)\right\}\right\}=\mathbf{P}\left\{\rho \in R\left(z_{2}\right)\right\} .
$$


Доказательство. Для любых $r \in R, z \in Z, s \in W_{j}, j=1, \ldots, m$, из (2) и (12) следует справедливость цепочек равенств

$$
\begin{aligned}
\psi\left(\varphi_{r}(\varphi(s))\right)=\psi(\varphi(\varphi(s))) & =\psi(\varphi(s)), \\
\psi\left(\varphi_{r}\left(\xi_{z}(s)\right)\right)=\psi\left(\varphi\left(\xi_{z}(s)\right)\right)=\psi\left(\xi_{z}\left(\xi_{z}(s)\right)\right)=\psi\left(\xi_{z}(s)\right) & =\psi(\varphi(s)) .
\end{aligned}
$$

Из леммы 1 следует, что $\xi_{z}\left(\xi_{z}(s)\right)$ и $\xi_{z}(s)$ лежат в $V_{j}$, а это означает совпадение двух крайних членов последних цепочек. Утверждения теоремы (14) и (15) доказаны.

Для каждого $z \in Z$ существует такое $R(z)$, что

$$
\xi_{z}(S)=\bigcup_{r \in R(z)} \varphi_{r}(S)
$$

и для любого подмножества $Q \subset R(z)$, не равного $R(z)$, объединение

$$
\bigcup_{r \in Q} \varphi_{r}(S)
$$

не включает $\xi_{z}(S)$. Если $R(z)=R$, то $\xi_{z}(S)=\varphi(S),|Z|=1$, и соотношения (16) и (17) доказаны.

Пусть существуют такие $r \in R, z_{1}, z_{2}$, что

$$
\varphi_{r}(S) \cap \xi_{z_{1}}(S) \neq \varnothing, \quad \varphi_{r}(S) \cap \xi_{z_{2}}(S) \neq \varnothing .
$$

Тогда в силу того, что разложение (13) является вложенным по отношению к разложению (6),

$$
\xi_{z_{1}}(S) \cap \xi_{z_{2}}(S) \subset \varphi_{r}(S)
$$

Это возможно лишь при $z_{1}=z_{2}$, что, в свою очередь, означает, что множества $R(z)$, не пересекаются при разных $z \in Z$. В силу (6) и (13) справедливо (16). А это означает, что

$$
\left\{r: \varphi_{r}\left(\xi_{z_{1}}(S)\right) \in \xi_{z_{2}}(S)\right\}=R\left(z_{2}\right) .
$$

Это влечет справедливость (17).

Теорема доказана.

Теорема 5. Пусть семейство расширений $\left\{\left(\varphi_{r}, \psi_{r}\right), r \in R\right\}$ является регулярным разложением протокола $(\varphi, \psi),\left\{\left(\xi_{z}, \eta_{z}\right), z \in Z\right\}-$ некоторое разложение протокола $(\varphi, \psi)$, отображение $\varphi$ удовлетворяет условию (12), и $\rho$-некоторая случайная величина, принимающая значения из $R$.

Тогда для любых $s \in S, z_{1}, z_{2} \in Z$ справедливо равенство

$$
\mathbf{P}\left\{\rho \in\left\{r: \varphi_{r}\left(\xi_{z_{1}}(s)\right) \in \xi_{z_{2}}(S)\right\}\right\}=\mathbf{P}\left\{\rho \in\left\{r: \xi_{z_{2}}(S) \cap \varphi_{r}(S) \cap \psi^{-1}(\psi(\varphi(s))) \neq \varnothing\right\}\right\} .
$$

Доказательство. Из соотношения (14) следует, что $\varphi_{r}\left(\xi_{z_{1}}(s)\right) \in \psi^{-1}(\psi(\varphi(s)))$ и, кроме того, $\varphi_{r}\left(\xi_{z_{1}}(s)\right) \in \varphi_{r}(S)$. В силу регулярности разложения

$$
\left|\varphi_{r}(S) \cap \psi^{-1}(\psi(\varphi(s)))\right|=1
$$

Это, наряду со сказанным выше, означает, что

$$
\varphi_{r}\left(\xi_{z_{1}}(s)\right)=\varphi_{r}(S) \cap \psi^{-1}(\psi(\varphi(\xi)))
$$


и в силу (18)

$$
\begin{aligned}
\left\{r: \varphi_{r}\left(\xi_{z_{1}}(s)\right) \in \xi_{z_{2}}(S)\right\} & =\left\{r: \varphi_{r}(S) \cap \psi^{-1}(\psi(\varphi(s))) \in \xi_{z_{2}}(S)\right\} \\
& =\left\{r: \xi_{z_{2}}(S) \cap \varphi_{r}(S) \cap \psi^{-1}(\varphi(s)) \neq \varnothing\right\}
\end{aligned}
$$

Последнее соотношение завершает доказательство теоремы.

Следствие 2. Пусть в условиях теоремы 5 случайная величина $\rho$ имеет равномерное распределение на $R$. Тогда справедливо соотночение

$$
\mathbf{P}\left\{\rho \in\left\{r: \varphi_{r}\left(\xi_{z_{1}}(s)\right) \in \xi_{z_{2}}(S)\right\}\right\}=\frac{|\psi(\varphi(S))|}{|\varphi(S)|}\left|\xi_{z_{1}}(S) \cap \psi^{-1}(\psi(\varphi(s)))\right|
$$

Если, кроме того $|\varphi(S)|=|S|$, то

$$
\frac{1}{|S|} \sum_{s \in S} \mathbf{P}\left\{\rho \in\left\{r: \varphi_{r}\left(\xi_{z_{1}}(s)\right) \in \xi_{z_{2}}(S)\right\}\right\}=\frac{\left|\xi_{z_{2}}(S)\right|}{|S|}
$$

Замечание 5. Пусть для защиты протокола $(\varphi, \psi)$, допускающего разложение (6), используется защита, основанная на разложении (13). Тогда смысл фигурирующих в теоремах 4 и 5 вероятностей заключается в следующем.

Решения, принимаемые участником не изменяются ни от действия защиты, ни при подмене протокола $(\varphi, \psi)$ на протоколы $\left(\xi_{z}, \eta_{z}\right), z \in Z$.

Вероятность решения сообщника $z_{2}$ не зависит от передаваемой ему команды злоумышленника $z_{1}$.

Замечание 6. Рассмотрим результаты применения описанной выше защиты к одному примеру построения процедуры скрытого общения злоумышленника с сообщником из [5].

Описание процедуры дается в терминах раздела 2.2 .

Пусть $S=\left(h_{0}, h_{1}\right), t_{0}-$ фиксированный элемент из $S$. Рассмотрим пару протоколов $\left(\xi_{0}, \eta_{0}\right),\left(\xi_{1}, \eta_{1}\right)$, в которых $\xi_{0}=\tau_{t_{0}}, \eta_{0}=\eta_{1}=\varepsilon, \xi_{1}-$ произвольное отображение множества $S$ на множество $S \backslash t_{0}$.

Тогда, по построению, определенная выше пара является разложением протокола $(\varepsilon, \chi)$, которое в силу первого утверждения теоремы 3 и замечания 3 является вложенным по отношению к разложению $\left\{\left(\tau_{t}, \varepsilon\right), t \in S\right\}$. Злоумышленник использует пару $\left(\xi_{0}, \eta_{0}\right)$, $\left(\xi_{1}, \eta_{1}\right)$ для передачи сообщнику команды на начало действия $t_{0}$.

Из теоремы 4 и замечания 5 при равномерном распределении $\rho$ на множестве $S$ следует, что вероятность правильного решения на начало действий равна

$$
\frac{h_{0} ! h_{1} !}{\left(h_{0}+h_{1}\right) !}
$$

Отсюда следует, что вероятность правильного решения в процедуре (активизации агента) [5], даже в том случае, когда он знает систему адресации сегментов и внутренних адресов, при применении описанной выше зашиты будет стремиться к 0 с ростом $h_{0}+h_{1}$.

Автор выражает признательность И. А. Круглову за внимание к работе и ряд конструктивных замечаний. В частности, им предложена лемма 1, которая существенно расширяет границы применимости теоремы 4. 


\section{Список литературы}

1. Шеннон К., Работы по теории информачии и кибернетике. ИЛ, Москва, 1963.

2. Князев А. В., Ронжин А. Ф., О простейших махинациях в некоторых системах нотариального заверивания цифровых подписей. Обозрение прикладной и промышленной математики (2003) 10, №2, 481-482.

3. Lampson B. W., A note of the confinement problem. Commun. ACM (1973) 16, №10, 613-615.

4. Schneier B., Applied cryptography: protocols, algorithms and source code in C. Wiley, New York, 1996.

5. Грушо А. А., Тимонина Е. Е., Оценка времени, требуемого для организации скрытого канала. Дискретная математика (2003) 15, №2, 40-46.

Статья поступила 23.03.2004. 